\title{
PRICKLE3 linked to ATPase biogenesis manifested Leber's hereditary optic neuropathy
}

\author{
Jialing Yu, ${ }^{1,2,3,4}$ Xiaoyang Liang, ${ }^{2,3}$ Yanchun Ji, ${ }^{1,2,3}$ Cheng Ai, ${ }^{2,3}$ Junxia Liu, ${ }^{2,3}$ Ling Zhu, ${ }^{1,2,3}$ Zhipeng Nie, ${ }^{2,3}$ Xiaofen Jin, ${ }^{2,3,4}$ \\ Chenghui Wang, ${ }^{2,3}$ Juanjuan Zhang, ${ }^{2,5}$ Fuxin Zhao, ${ }^{5}$ Shuang Mei, ${ }^{2,3}$ Xiaoxu Zhao, ${ }^{1,2,3}$ Xiangtian Zhou, ${ }^{5}$ Minglian Zhang, ${ }^{6}$ \\ Meng Wang, ${ }^{1,2,3}$ Taosheng Huang, ${ }^{7}$ Pingping Jiang, ${ }^{1,2,3}$ and Min-Xin Guan ${ }^{1,2,3,4,8}$ \\ 'Division of Medical Genetics and Genomics, Children's Hospital, Zhejiang University School of Medicine and National Clinical Research Center for Child Health, Hangzhou, China. ${ }^{2}$ Institute of Cenetics and \\ ${ }^{3}$ Department of Human Genetics, Zhejiang University School of Medicine, Hangzhou, China. ${ }^{4}$ Key Laboratory of Reproductive Genetics, Ministry of Education, Hangzhou, China. ${ }^{5}$ School of Optometry and \\ Ophthalmology and Eye Hospital, Wenzhou Medical University, Wenzhou, China. ${ }^{6}$ Department of Ophthalmology, Hebei Provincial Eye Hospital, Xingtai, China. ${ }^{7}$ Division of Human Genetics, Cincinnati \\ Children's Hospital Medical Center, Cincinnati, Ohio, USA. ${ }^{8}$ Joint Institute of Cenetics and Genomic Medicine, Zhejiang University and University of Toronto, Zhejiang University, Hangzhou, China.
}

\begin{abstract}
Leber's hereditary optic neuropathy (LHON) is a maternally inherited eye disease. X-linked nuclear modifiers were proposed to modify the phenotypic manifestation of LHON-associated mitochondrial DNA (mtDNA) mutations. By whole-exome sequencing, we identified the X-linked LHON modifier (c.157C >T, p.Arg53Trp) in PRICKLE3 encoding a mitochondrial protein linked to biogenesis of ATPase in 3 Chinese families. All affected individuals carried both ND4 11778G>A and p.Arg53Trp mutations, while subjects bearing only a single mutation exhibited normal vision. The cells carrying the p.Arg53Trp mutation exhibited defective assembly, stability, and function of ATP synthase, verified by PRICKLE3-knockdown cells. Coimmunoprecipitation indicated the direct interaction of PRICKLE3 with ATP synthase via ATP8. Strikingly, cells bearing both p.Arg53Trp and $\mathrm{m} .11778 \mathrm{C}>\mathrm{A}$ mutations displayed greater mitochondrial dysfunction than those carrying only a single mutation. This finding indicated that the p.Arg53Trp mutation acted in synergy with the $\mathrm{m} .11778 \mathrm{G}>\mathrm{A}$ mutation and deteriorated mitochondrial dysfunctions necessary for the expression of LHON. Furthermore, we demonstrated that Prickle3deficient mice exhibited pronounced ATPase deficiencies. Prickle3-knockout mice recapitulated LHON phenotypes with retinal deficiencies, including degeneration of retinal ganglion cells and abnormal vasculature. Our findings provided new insights into the pathophysiology of LHON that were manifested by interaction between mtDNA mutations and X-linked nuclear modifiers.
\end{abstract}

\section{Introduction}

Leber's hereditary optic neuropathy (LHON) is the most common maternally inherited eye disease (1). This disorder is characterized by bilateral, painless, subacute, central visual loss in young adults $(2,3)$. The typical features in this disorder include the primary degeneration of retinal ganglion cells (RGCs) accompanied by ascending optic atrophy $(4,5)$. Mutations in mitochondrial DNA (mtDNA) have been identified to contribute to the pathogenesis of LHON though to varying degrees (6-10). Of these, the m.11778G $>$ A mutation in the ND4 gene encoding a subunit of NADH:ubiquinone oxidoreductase (complex I) is the most prevalent LHON-associated mtDNA mutation worldwide (7-12). However, only relatively mild mitochondrial dysfunctions, especially the reduced activity of complex I, were observed in cells carrying the $\mathrm{m} .11778 \mathrm{G}>\mathrm{A}$ mutation $(13-16)$. There were marked variations in the penetrance and sex bias occurring in the LHON pedigrees carrying the mtDNA mutation, reflecting the complex etiology of

Authorship note: JY, XL, YJ, and CA equally contributed to the work Conflict of interest: The authors have declared that no conflict of interest exists. Copyright: (5) 2020, American Society for Clinical Investigation. Submitted: November 14, 2019; Accepted: June 4, 2020; Published: August 10, 2020 Reference information: J Clin Invest. 2020;130(9):4935-4946. https://doi.org/10.1172/JCl134965. this disease $(1,5,17,18)$. Our recent study demonstrated that several families with LHON manifested synergic interaction between m.11778G $>$ A mutation and mutated autosomal recessive modifier YARS2 encoding mitochondrial tyrosyl-tRNA synthetase (15). The incomplete penetrance and sex bias in patients presenting with optic neuropathy suggest an X-linked modifier gene necessary for the phenotypic expression of LHON-associated mtDNA mutations (19). Despite statistical support for the linkages of several putative nuclear modifier loci in the $\mathrm{X}$ chromosome, the exact gene has yet to be identified $(20,21)$.

In this study, we performed whole-exome sequencing of genetically uncharacterized patients with LHON by taking advantage of a large cohort of 1281 Chinese probands with LHON (11, $22,23)$. As a result, we identified a potentially novel LHON susceptibility allele (c.157C > T, p.Arg53Trp) in PRICKLE3 at Xp11.23, which is located at the putative nuclear modifier loci for the phenotypic expression of LHON-associated mtDNA mutations $(20,21)$. PRICKLE3 encodes a highly conserved protein enriched in mitochondria, whose Drosophila and Xenopus homologs are involved in planar cell polarity and vision function (24-26). We then performed Sanger sequencing analysis of symptomatic and asymptomatic individuals among 211 Chinese and 58 Caucasian pedigrees carrying the m.11778G $>$ A mutation and 1515 Chinese and 237 Caucasian control subjects lacking the m.11778G $>$ A mutation. 
Functional significance of the PRICKLE3 mutation was assessed through the use of lymphoblastoid mutant cell lines derived from matrilineal relatives of Chinese families (individuals carrying only the $\mathrm{m} .11778 \mathrm{G}>\mathrm{A}$ mutation or both $\mathrm{m} .11778 \mathrm{G}>\mathrm{A}$ and heterozygous or hemizygous PRICKLE3 mutations), normal-vision individuals carrying only PRICKLE3 mutation, and genetically unrelated control subjects lacking these mutations. The effect of p.Arg53Trp mutation on the oxidative phosphorylation system (OXPHOS) was further evaluated for the activity and assembly of mitochondrial ATPase using PRICKLE3 knockdown in HeLa cells by shRNA. To investigate whether defects in PRICKLE3 cause the degeneration of RGCs in vivo, we studied Prickle3-knockout mice produced by genome editing using the CRISPR/Cas9 system.

\section{Results}

Identification of PRICKLE3 mutation by whole-exome sequencing. We performed whole-exome sequencing of DNA from 4 members (proband III-14, affected mother II-8, unaffected sibling III-15, and father II-7) of the SD1 family carrying the m.11778G>A mutation (Figure 1A, Supplemental Table 1, and ref. 27; supplemental material available online with this article; https://doi.org/10.1172/ JCI134965DS1). The overview of the exome analysis is summarized in Supplemental Figure 1 and Supplemental Table 2. After removing annotated polymorphisms and filtering for variants, a single exonic variant (c.157C>T) (chromosome X: 49040342 $\mathrm{C}>\mathrm{G}$, hg 19) in exon 3 of the PRICKLE3 gene was identified at $\mathrm{Xp11.23}$, which localized at the putative modifier region predicted to manifest the phenotypic expression of $\operatorname{LHON}(20,21)$. The c. $157 \mathrm{C}>\mathrm{T}$ mutation changed a highly conserved 53 arginine with tryptophan (p.Arg53Trp) at the N-terminal of PRICKLE3 (Figure $1 \mathrm{~B})$. PRICKLE3 is a highly conserved protein from vertebrates to primates (Supplemental Figure 2), belonging to the PRICKLE family involved in planar cell polarity necessary for the development of retinas $(26,28)$.

We then carried out Sanger sequencing analysis of DNA fragments spanning all exons and their flanking sequences of PRICKLE3 among 35 members of this family (Supplemental Table 3). Of 21 matrilineal relatives, 5 symptomatic men carried the hemizygous c.157C $>$ T mutation, 9 symptomatic women harbored the heterozygous c.157C $>$ T mutation, and 7 (4 women and 3 men) asymptomatic subjects lacked the c.157C $>$ T mutation. No other sequence changes were detected among these individuals. Of other family members lacking the m.11778G $>$ A mutation, only 1 woman (IV-3) carried the heterozygous c. $157 \mathrm{C}>\mathrm{T}$ mutation; the remaining individuals lacked the c.157C $>\mathrm{T}$ mutation. We further analyzed the presence of the c.157C>T mutation in 345 symptomatic and 234 asymptomatic subjects from 210 Han Chinese pedigrees, 58 symptomatic and 58 asymptomatic subjects from 58 Caucasian pedigrees carrying the m.11778G>A mutation, and 1515 (815 men and 700 women) Han Chinese and 237 (137 men and 100 women) Caucasian control subjects lacking the $\mathrm{m} .11778 \mathrm{G}>\mathrm{A}$ mutation. Four men and 5 women, symptomatic subjects belonging to pedigrees $\mathrm{XT}$ and $\mathrm{AH}$, harbored the hemizygous or heterozygous c.157C $>\mathrm{T}$ mutation, respectively. The c.157C $>$ T mutation was absent among 234 Chinese asymptomatic subjects and 116 Caucasian subjects bearing the m.11778G $>$ A mutation and 237 controls. The c. $157 \mathrm{C}>\mathrm{T}$ mutation was present only in hemizygous form in 2 male (XT-II-3,
AH-I-1) and in heterozygous form in 1 female (XT-III-6) married-in control subjects lacking the m.11778G >A mutation among 1515 Chinese control subjects. Furthermore, no other functionally significant mtDNA variants were found in these families, suggesting that mtDNA backgrounds may not play an important role in the phenotypic manifestation of m.11778G $>$ A mutation (Supplemental Table 4 and ref. 29). The cosegregation of LHON phenotype with the presence of both $\mathrm{m} .11778 \mathrm{G}>\mathrm{A}$ and $\mathrm{c} .157 \mathrm{C}>\mathrm{T}$ mutations in 9 men and 14 women suggested that the $c .157 \mathrm{C}>\mathrm{T}$ mutation is a rare X-linked dominant LHON-susceptible allele.

The p.Arg53Trp mutation affected the stability but not mitochondrial localization of PRICKLE3. To investigate the effect of the p.Arg53Trp mutation on PRICKLE3, we examined the level of PRICKLE3 protein by Western blot analysis using mutant and control lymphoblastoid cell lines derived from members of the SD1 pedigree. As illustrated in Figure 1C, the cell lines carrying the p.Arg53Trp mutation, with or without the $\mathrm{m} .11778 \mathrm{G}>\mathrm{A}$ mutation, exhibited an approximately $55 \%$ reduction in the levels of PRICKLE3 and no effect on the expression levels of PRICKLE1 and PRICKLE4, indicating a deleterious effect of p.Arg53Trp mutation on PRICKLE3 structure/function.

Potential mitochondrial dysfunction caused by the p.Arg53Trp mutation led to us to examine whether PRICKLE3 localizes in mitochondria. The Mitoprot program predicted that PRICKLE3 has a 0.3314 probability to enter into mitochondria with a 15-amino acid sequence targeting mitochondria (30). To determine the mitochondrial localization of PRICKLE3, an HA-tagged version of PRICKLE3 was transiently expressed within the HeLa cells. Cellular fraction experiments of HeLa cells revealed that the exogenous PRICKLE3 was enriched within mitochondrial fractions, along with outer mitochondrial membrane protein TOMM2O and inner mitochondrial membrane protein ATP5F, and present in cytosol, along with the cytosolic protein tubulin (Figure 1D). This indicates that mitochondria contain the PRICKLE3 protein. Furthermore, a light band of PRICKLE3 in isolated mitochondria was resistant to treatment with proteinase K, implying that PRICKLE3 may be recruited to the inner membrane for interaction or binding with protein(s) that anchored in the inner membrane. Moreover, a carboxy terminus HA-tagged WT or mutant PRICKLE3 displayed overlap with the mitochondrial protein UQCRC2, with a significant correlation (Pearson coefficient of 0.61 and 0.55 for WT and mutant, respectively) between red- and green-colored channels (Figure 1E). These results indicated that PRICKLE3 localized to mitochondria but that the p.Arg53Trp mutation did not affect its mitochondrial location.

The PRICKLE3 mutation caused defects in mitochondrial complex $V$ function. To evaluate the effect of the p.Arg53Trp mutation on oxidative phosphorylation, we measured the activities of respiratory complexes from cell lines derived from subjects carrying only m.11778G >A or p.Arg53Trp mutation and both m.11778G >A with heterozygous or hemizygous p.Arg53Trp mutations. As shown in Figure 2A, the activity of complex I in the mutant cell lines carrying only $\mathrm{m} .11778 \mathrm{G}>\mathrm{A}$ mutation, only p.Arg53Trp mutation, and both $\mathrm{m} .11778 \mathrm{G}>\mathrm{A}$ and heterozygous or hemizygous p.Arg53Trp mutations was $77 \%, 94 \%, 65 \%$, and $62 \%$ of the average values in control cell lines lacking these mutations, respectively. However, the activities of complexes II, III, and IV in all mutant cell lines 

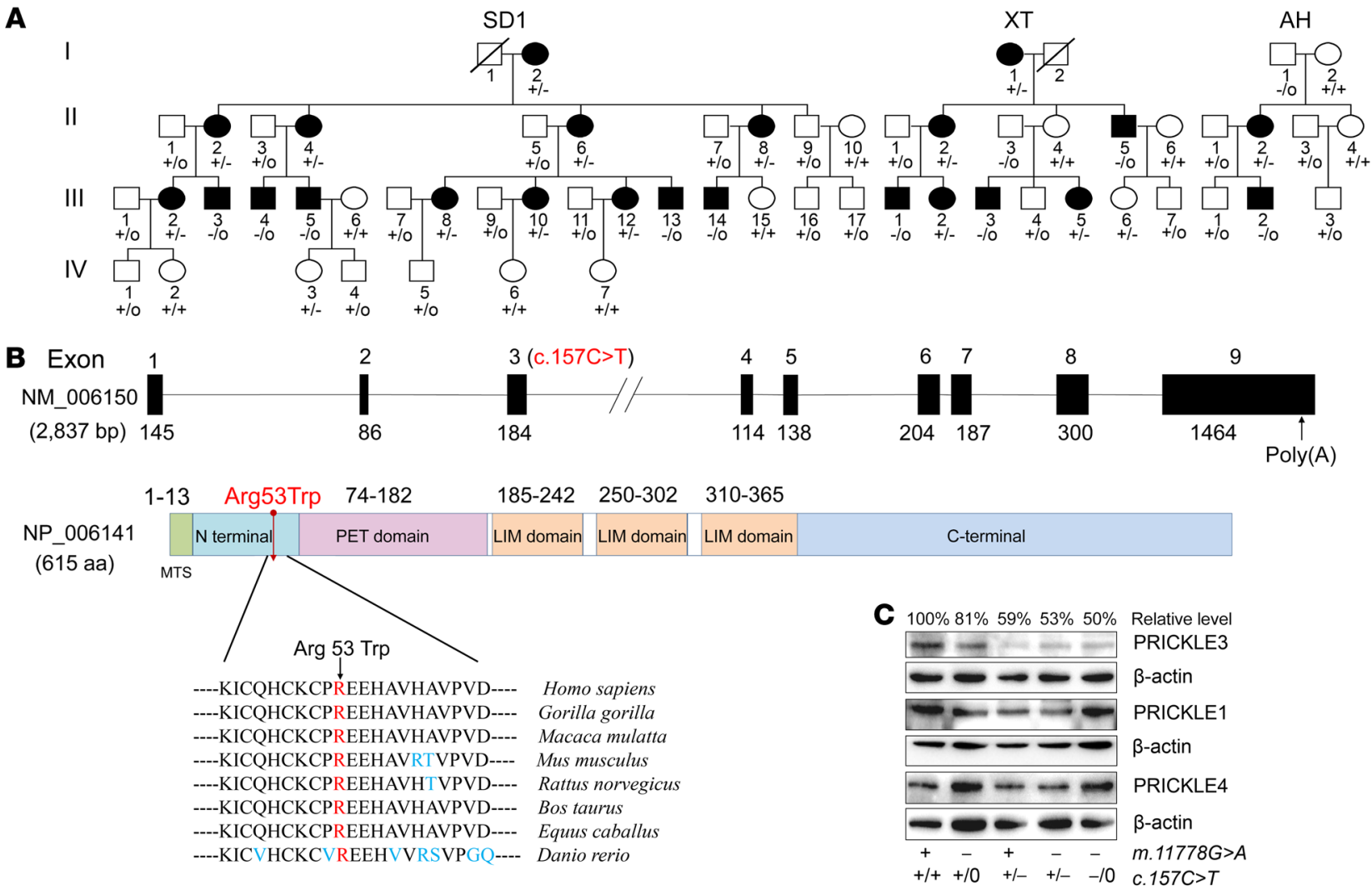

D

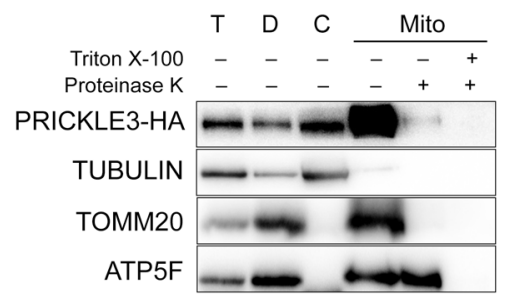

$\mathbf{E}$

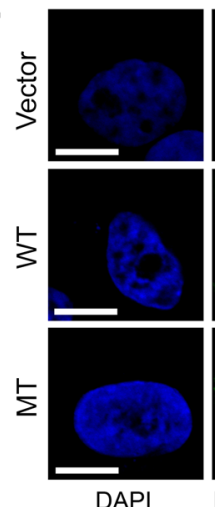

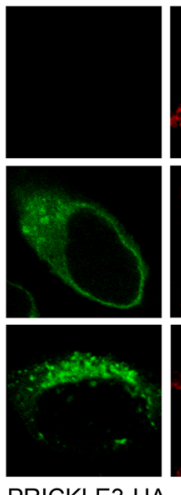

PRICKLE3-HA

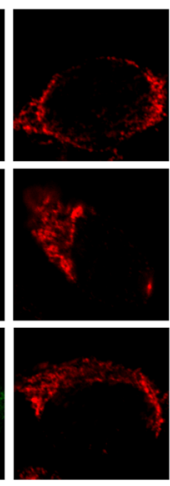

UQCRC2

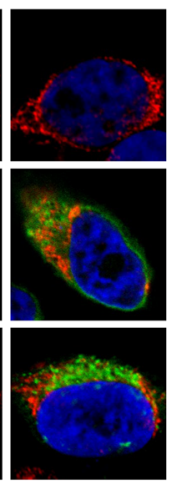

Merge

Figure 1. Identification of PRICKLE3 p.Arg53Trp mutation. (A) Three Chinese pedigrees with LHON. Vision-impaired individuals are indicated by blackened symbols. Individuals harboring hemizygous (-/0) or heterozygous (+/-) PRICKLE3 (c.157C>T, p.Arg53Trp) mutation and WT (+/+ or +/0) are indicated. (B) Scheme for the structure of PRICKLE3 and its product, multiple sequence alignment of homologs. (C) Western blot analysis of PRICKLE3, PRICKLE1, and PRICKLE4 in various lymphoblastoid cell lines. The levels of these proteins were quantified in the linear range of the sample loading and expressed as percentages of the average values for the control cell lines. (D) Subcellular localization of PRICKLE3 by Western blotting with anti-HA, TOMM2O (outer mitochondrial membrane), ATP5F (inner mitochondrial membrane), and tubulin (cytosol). T, total cell lysate; D, debris; C, cytosol; Mito, mitochondria. Isolated mitochondria were treated with (+) or without (-) 1\% Triton X-100 followed by proteinase K digestion, respectively. (E) Subcellular localization of PRICKLE3 by immunofluorescence in HeLa cells. HA-PRICKLE3 WT or mutant (MT) (shown in green), UQCRC2 (shown in red), and DAPI (shown in blue). Scale bar: $10 \mu \mathrm{m}$. See complete unedited blots in the supplemental material.

were comparable with those of control cell lines. These suggested that the $\mathrm{m} .11778 \mathrm{G}>\mathrm{A}$ mutation contributed to the reduced activity of complex I but that the p.Arg53Trp mutation did not affect the activities of those complexes (Figure 2A). Strikingly, the activity of complex $\mathrm{V}$ in the mutant cell carrying only $\mathrm{m} .11778 \mathrm{G}>\mathrm{A}$ mutation, only p.Arg53Trp mutation, and both $\mathrm{m} .11778 \mathrm{G}>\mathrm{A}$ and heterozygous or hemizygous p.Arg53Trp mutations was 87\%, 56\%, 49\%, and $32 \%$ of the average values in control cell lines, respectively.
The reduced activities of complex $\mathrm{V}$ in various mutant cells were further confirmed by clear native polyacrylamide gel electrophoresis, supplied with mitochondrial ATPase substrates, reflecting $82 \%, 51 \%, 45 \%$, and $41 \%$ of the average values in controls (Figure 2B and refs. 31, 32). Moreover, the $\mathrm{F}_{1}$ signal was markedly reduced among the different mutant cell lines. These data revealed that the p.Arg53Trp altered the activity of complex $\mathrm{V}$ but did not perturb the activities of other respiratory chain complexes. 
A

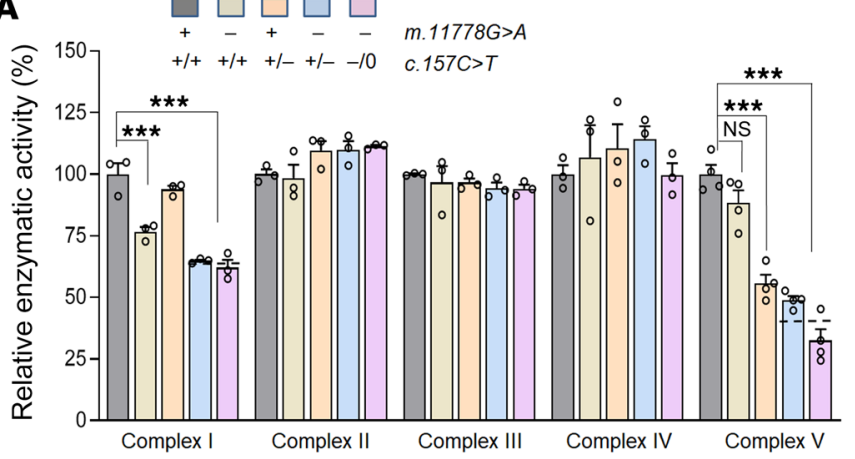

B

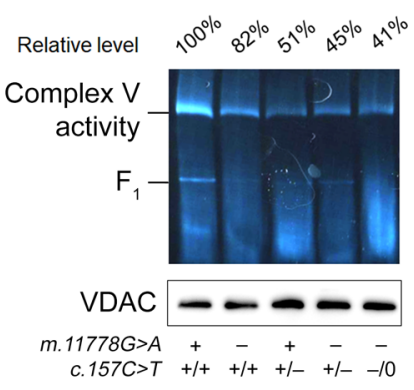

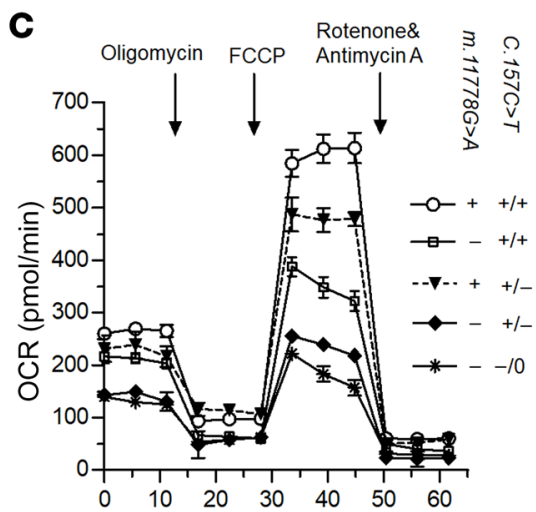
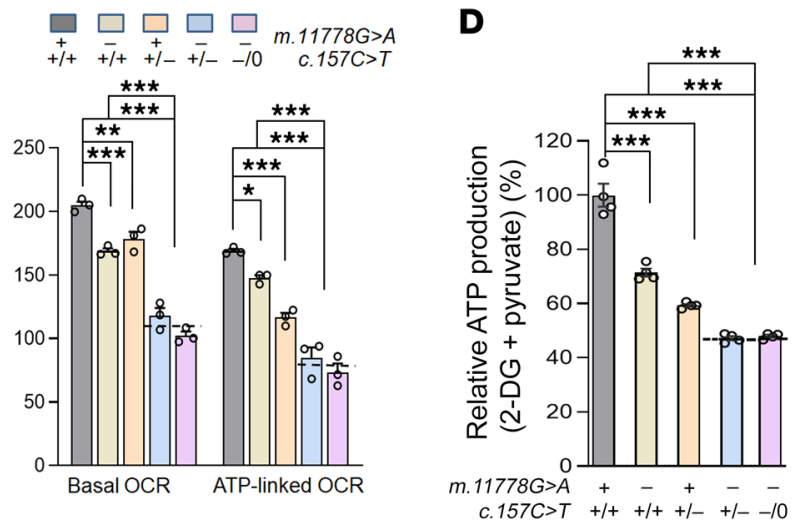

Figure 2. The p.Arg53Trp mutation caused the defective activity of mitochondrial ATPase. (A) Enzymatic activities of respiratory chain complexes in mitochondria isolated from various cell lines. (B) In-gel activity of ATPase. (C) The rates of OCR $\left(\mathrm{O}_{2}\right.$ consumption) in the various cell lines using different inhibitors. Basal OCR was determined as OCR before oligomycin minus OCR after rotenone/antimycin A. ATP-lined OCR was determined as OCR before oligomycin minus OCR after oligomycin. (D) Measurement of mitochondrial ATP levels using a bioluminescence assay. Cells were incubated with 5 mM 2-deoxy-D-glucose plus $5 \mathrm{mM}$ pyruvate to determine ATP generation under mitochondrial ATP synthesis. Average rates of ATP level per cell line and are shown. Dashed lines indicate the mean values of enzymatic activities (A), OCRs (C), or ATP production (D) in PRICKLE3 $3^{+/-}$and $P R I C K L E 3^{-/ 0}$ cell lines. Data are shown as mean \pm SEM. ${ }^{*} P<0.05 ;{ }^{* *} P<0.01 ;{ }^{* *} P<0.001$ by 2 -way ANOVA (A and $\mathbf{C}$ ) and 1-way ANOVA (D) followed by Bonferroni's post hoc test; NS, not significant. Representative of 3 to 4 independent experiments.

To validate whether the defective complex $\mathrm{V}$ activity was caused by the p.Arg53Trp mutation, we measured the oxygen consumption rates (OCRs) in various mutant and control cell lines. As shown in Figure 2C, cell lines carrying only p.Arg53Trp or $\mathrm{m} .11778 \mathrm{G}>\mathrm{A}$ mutation displayed mild reductions in the basal OCR and ATP-linked OCR, while more drastic reductions in these OCRs were observed in cell lines carrying both $\mathrm{m} .11778 \mathrm{G}>\mathrm{A}$ and p.Arg53Trp mutations. However, the PRICKLE3 mutation did not affect the levels of 6 subunits of the OXPHOS: NDUFA9, NDUFB8, SDHA, UQCRC2, CO2, and ATP5A (Supplemental Figure 3). To investigate whether the mitochondrial contents reflect these biochemical data, we analyzed the mtDNA copy numbers in the mutant and control cell lines. No significant differences in the contents of mtDNA were detected among mutant and control cell lines (Supplemental Figure 4). Using luciferin/luciferase assay for measurement of ATP production, a 53\% reduction in the level of mitochondrial ATP was observed in cell lines carrying both m.11778G $>$ A and p.Arg53Trp mutations, while only $28.6 \%$ and $40.7 \%$ decreases in the level of mitochondrial ATP were observed in the cell lines carrying only m.11778G $>A$ or p.Arg53Trp mutation, respectively (Figure 2D). These observations suggested that the p.Arg53Trp mutation worsened the complex V deficiency caused by the m.11778G $>$ A mutation. However, the p.Arg53Trp mutation did not affect the levels of reactive oxygen species or mitochondrial membrane potentials (Supplemental Figure 5).

The PRICKLE3 mutation altered the stability of complex $V$. To investigate whether the deficient ATP production results from the altered assembly or instability of mitochondrial ATP synthase, which consists of $\mathrm{F}_{\mathrm{O}}$ (c-ring and a, b, d, e, f, g, and A6L subunits) and $\mathrm{F}_{1}(\alpha, \beta, \gamma, \delta$, and $\varepsilon$ subunits) regions $(33,34)$, mitochondrial membrane proteins from mutant and control cell lines were separated by blue native polyacrylamide gel electrophoresis and hybridized with ATP5A antibody (a nuclear encoded complex V subunit) and VDAC as the loading control. As illustrated in Figure 3A, the levels of complex $\mathrm{V}$ monomer in mutant cell lines carrying only m.11778G $>\mathrm{A}$ mutation, only p.Arg53Trp mutation, and both $\mathrm{m} .11778 \mathrm{G}>\mathrm{A}$ and heterozygous or hemizygous p.Arg53Trp mutations were $95 \%, 58 \%, 55 \%$, and $59 \%$ of the mean values in control cell lines, respectively. These results were validated by an approximately $31 \%$ decrease of fully assembled complex $\mathrm{V}$ observed in the PRICKLE3-silenced HeLa cells and rescued by overexpression of PRICKLE3 (Figure 3B, Supplemental Figure 6). We then measured the levels of ATP6 (a), ATP8 (A6L), ATP5F (b), ATP5A $(\alpha)$, ATP5B ( $\beta)$, and ATP5C $(\gamma)$ subunits of ATP synthase 
A

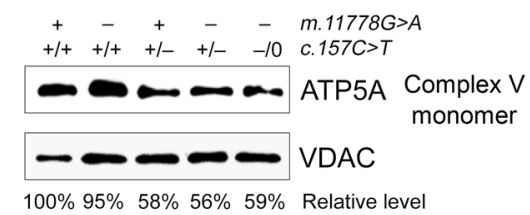

B $100 \% 69 \% \quad 86 \%$ Relative level
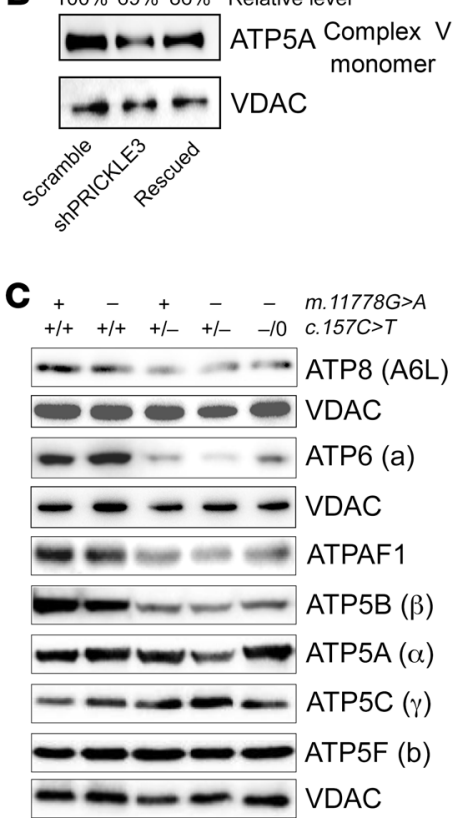

D

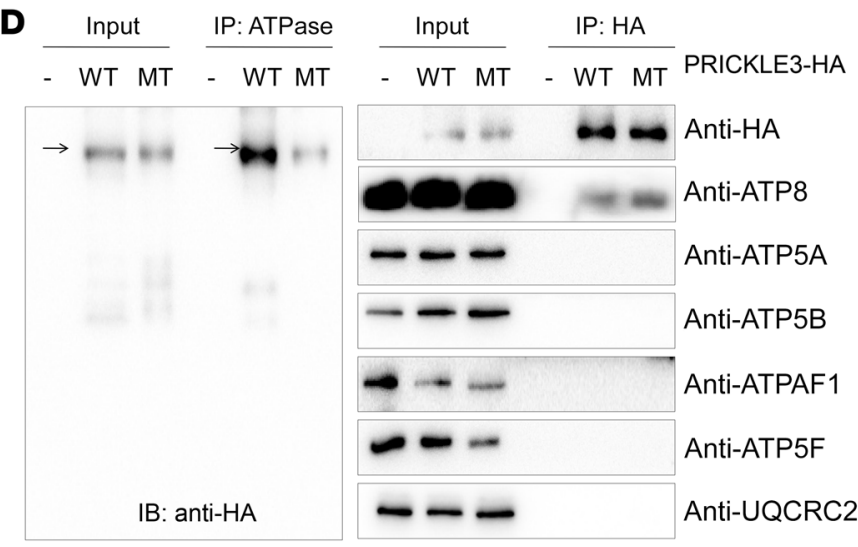

$\mathbf{E}$

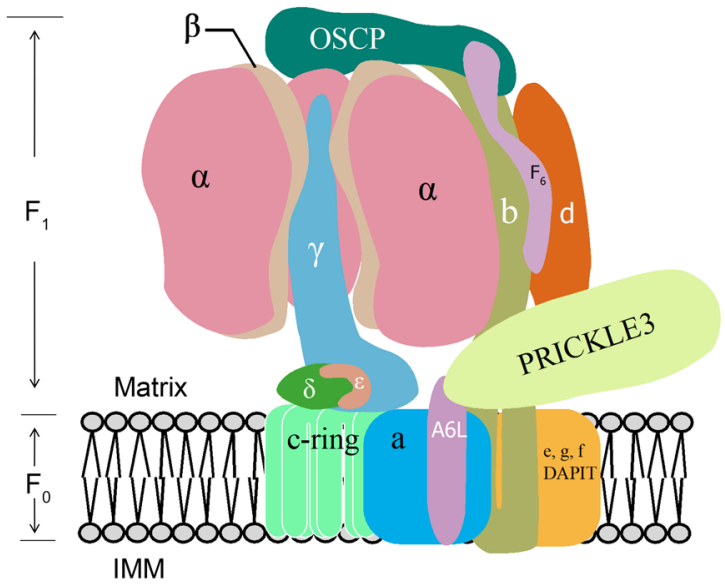

Figure 3. The p.Arg53Trp mutation altered the assembly and stability of ATP synthase. (A and B) The stability of fully assembled ATP synthase monomer. Mitochondria isolated from various lymphoblastoid cell lines (A) and HeLa cells, which were transfected by shRNA (PRICKLE3 and scramble) and rescued by WT-PRICKLE3 (B), subjected to blue native PAGE, and hybridized with anti-ATP5A antibody and VDAC as an internal control. Data are shown as mean \pm SEM of triplicates. (C) Immunoblot analysis of subunits of ATP synthase. Total cellular proteins in various lymphoblastoid cell lines were electrophoresed with PACE and hybridized with ATP6, ATP8, ATPAF1, ATP5B, ATP5A, ATP5C, and ATP5F antibodies and VDAC as a loading control, respectively. (D) Physical interaction of PRICKLE3 with ATP8 (A6L). Isolated mitochondria from HEK293T cells, transiently expressing WT, MT PRICKLE3-HA, and empty vector, were solubilized with $0.5 \%$ DDM. Lysate proteins were immunoprecipitated with ATP synthase immunocapture kit (left), HA antibody (right), respectively. Antibodies: anti-HA and anti-ATP8, -ATP5A, -ATP5B, -ATP5F, and -ATPAF1 for complex V and anti-UQCRC2 for complex III were used, respectively. (E) Proposed model for the direct interaction between PRICKLE3 and ATPase $\left(F_{0}: a, b, 8 / A 6 L, d, e, f, g\right.$, diabetes-associated protein in insulin-sensitive tissue; $F_{1}: \alpha, \beta, \gamma, \delta$, ع). Matrix, mitochondrial matrix; IMM, inner mitochondrial membranes. $F_{0} F_{1}$ ATP structural model was modified from He et al. (37).

and ATPAF1 involved in complex $\mathrm{V}$ assembly among mutant and control cell lines. Strikingly, the levels of ATP6, ATP8, ATPAF1, and ATP5B were markedly decreased in mutant cell lines carrying the p.Arg53Trp mutation, while there were no significant differences in the levels of other subunits between mutant and control cell lines (Figure 3C). In fact, ATPAF1 binds specifically to ATP5B $(\beta)$ as an assembly factor for $F_{1}(35)$, and ATP8 binds with ATP6 as a stator for the assembly of the peripheral stalk and $\mathrm{F}_{1}$ module (36, 37). This implied the possibility of a specific effect caused by the PRICKLE3 defect on the assembly of $\mathrm{F}_{1}$.

To examine whether PRICKLE3 directly interacts with mitochondrial ATP synthase, we performed an immunoprecipitation assay using ATP Synthase Immunocapture Kit and HA antibody in mitochondria of HEK293T cell lines overexpressed with WT and mutated forms of HA-tagged PRICKLE3. As shown in Figure 3D, PRICKLE3 and ATP synthase or ATP8 (A6L) reciprocally immunoprecipitated, respectively. By contrast, PRICKLE3 did not precipitate ATP5A ( $\alpha$ ), ATP5B ( $\beta$ ), ATP5F (b), ATPAF1, or UQCRC2.
These data demonstrated the interaction of PRICKLE3 with ATPase by specifically binding to ATP8 (A6L) (Figure 3E).

Prickle3-knockout mice exhibited deficient function of complex $V$. To investigate whether defects in PRICKLE3 cause the dysfunction of visual systems in vivo, we studied Prickle3-knockout (Prickle3-KO) mice produced by the CRISPR/Cas9 system (Figure 4A and Supplemental Figure 7A). This deletion caused a frameshift mutation resulting in a truncated Prickle3 protein with 61 amino acids (p.E61*) (Figure 4A). Both Western blot and immunohistochemistry data showed that Prickle3 was markedly reduced in the retinas of Prickle3-KO mice (Figure 4, B and C). All Prickle3+--, Prickle $^{-/ 0}$, and Prickle ${ }^{-/-}$mice were viable. Moreover, Prickle3 was ubiquitously expressed in various layers of retina and other mouse tissues (Supplemental Figure 7B). We then tested whether the deletion of Prickle 3 caused mitochondrial dysfunction in vivo. Prickle3 indeed localizes in mitochondria of mouse embryonic fibroblast cells (Supplemental Figure 7C). The enzymatic assays revealed $52 \%$ and $67 \%$ decreases in the activities of complex $\mathrm{V}$ in the retinas 
A

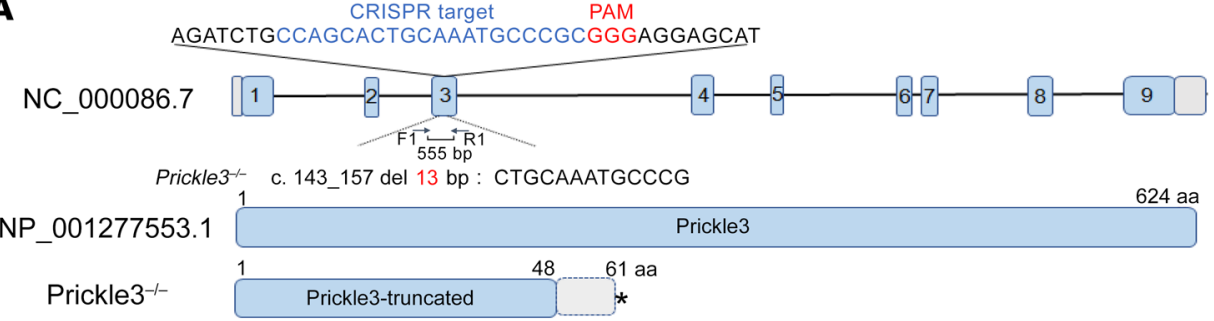

B
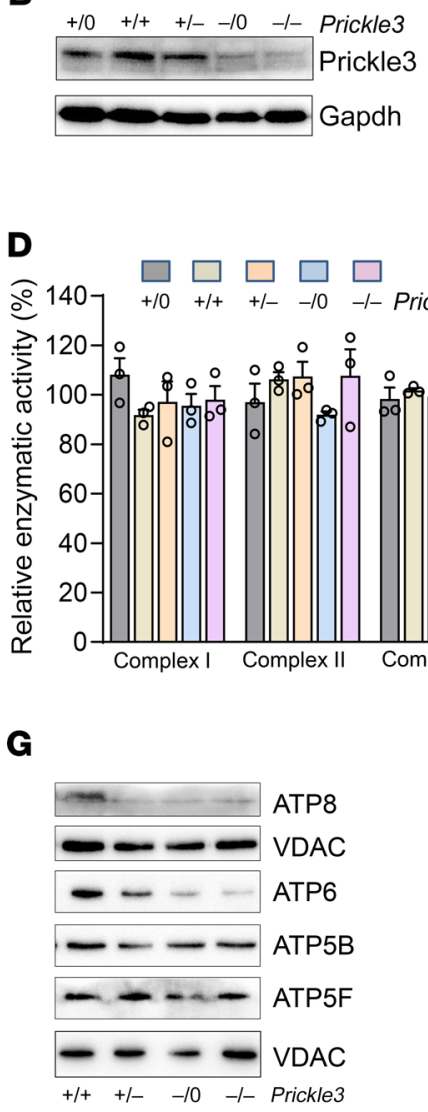

C

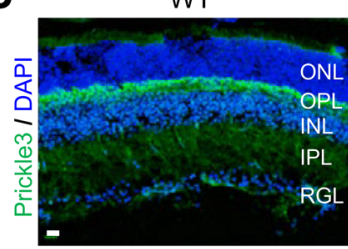

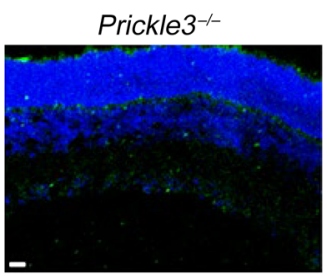

E
$\mathrm{V}_{\mathrm{M}}$

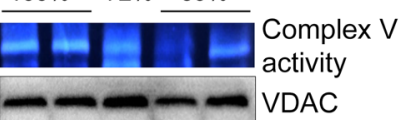

F

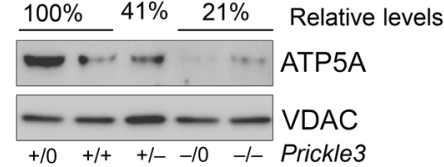

Figure 4. The altered activity and assembly of complex $V$ in retinas from Prickle3-KO mice. (A) Schema of generation of Prickle3-KO mice (C57BL/6]) using the CRISPR/ Cas9 system. A 13-bp deletion was produced in exon 3 (c.144_158del) of Prickle3, resulting in a truncated protein with 60 amino acids. (B) Levels of Prickle3 in retinas from different genotypes of mice at 8 weeks of age. (C) Prickle3 expression in retinas of WT and Prickle3-/- mice by immunolabeling analysis. ONL, outer nuclear layer; $\mathrm{OPL}$, outer plexiform layer; INL, inner nuclear layer; IPL, inner plexiform layer; RGL, ganglion cell layer. Scale bar: $10 \mu \mathrm{m}$. (D) Enzymatic activities of complexes I, II, III, IV, and $V$ in mitochondria isolated from the livers of Prickle3-KO and WT mice. Dashed lines indicate the mean values of enzymatic activities in samples of Prickle $3^{-10}$ and Prickle3 ${ }^{-1-}$ mice. Data are shown as mean \pm SEM of triplicates. ${ }^{* *} P<0.001$ by 2 -way ANOVA followed by Bonferroni's post hoc test. (E) In-gel activity of ATPase in the livers of Prickle3-KO and WT mice. $\mathrm{V}_{\mathrm{M}}$, complex $\mathrm{V}$ monomer. (F) Assembly of ATP synthase monomer using blue native PACE analysis in the liver mitochondria of Prickle3-KO and WT mice. (C) Levels of ATP8, ATP6, ATP5A, and ATP5F in the retinas of Prickle3-KO and WT mice. (H) Mitochondrial morphology in RGCs by transmission electron microscopy. Arrows indicate mitochondria. Scale bars: $1 \mu \mathrm{m}$ (upper panel), $0.2 \mu \mathrm{m}$ (lower panel).

of Prickle $^{+/-}$and Prickle $3^{-/ 0} /$ Prickle3 $^{-/-}$mice, respectively (Figure 4D). The reduced activities of ATP synthase in the Prickle $3^{-/ 0}$, Prickle $3^{-/}$, and Prickle $3^{+/-}$mice were further confirmed by blue native polyacrylamide gel electrophoresis (Figure 4E). The altered assembly of complex V caused by deletion of Prickle3 was evidenced by the $59 \%$ and $79 \%$ decreases of fully assembled ATP synthase in the Prickle3 $^{+/-}$and Prickle3 $3^{-/ 0} /$ Prickle3 $^{-/-}$mice, respectively, as compared with WT mice (Figure 4F). Furthermore, Prickle3-KO mice exhibited various decreases in the levels of ATP8, ATP6, and ATP5B, as compared with the WT mice (Figure 4G). Moreover, the retinas of Prickle $3^{+/}$and Prickle $^{-/-}$mice exhibited abnormal mitochondrial morphology, including vacuolated, fragmented mitochondria and the loss of cristae (Figure $4 \mathrm{H}$ ). These demonstrated that the deletion of Prickle 3 altered the function of mitochondrial ATPase. We concluded that the Prickle3-KO mice recapitulated the biochemical phenotypes in the patients with LHON.

Prickle3-KO mice exhibited retinal defects. We then investigated whether the loss of Prickle3 led to the typical phenotypes of LHON, including the degeneration of RGCs and vascular abnormalities. Retinal functions from Prickle $3^{-/}$, Prickle $3^{-/ 0}$, Prickle $3^{+-}$, and WT mice were first assessed by full-field electroretinography (ffERG), which measures widespread alteration of the functional integrity of the outer layer of the retina, including photoreceptors and bipolar cells (38). The Prickle $3^{-/}$, Prickle $3^{-/ 0}$, and Prickle ${ }^{+/-}$mice developed retinal deficiency at 4 weeks of age.

H\&E-stained retinal sections showed that the deletion of Prickle 3 did not change the thickness of layers in retinas of mice $(8$ weeks old) but caused the degeneration of RGCs (Figure 5A). To test whether the deletion of Prickle3 resulted in the loss of RGCs, the RGC layers from KO and WT mice were immunostained with Brn3a, $\beta$-III-tubulin antibodies, and DAPI to show nuclei $(39,40)$. Importantly, the RGCs (Brn3a-positive staining) in the retinal ganglion layers of Prickle $^{+/-}$and Prickle $3^{-/-}$mice were reduced by $22 \%$ and $39 \%$, as compared with WT littermates, respectively (Figure $5 \mathrm{~B})$. These results demonstrated that the ablation of Prickle 3 caused the loss of RGCs. 
A

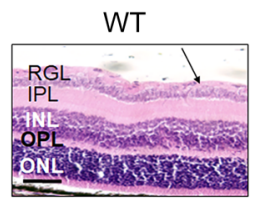

B
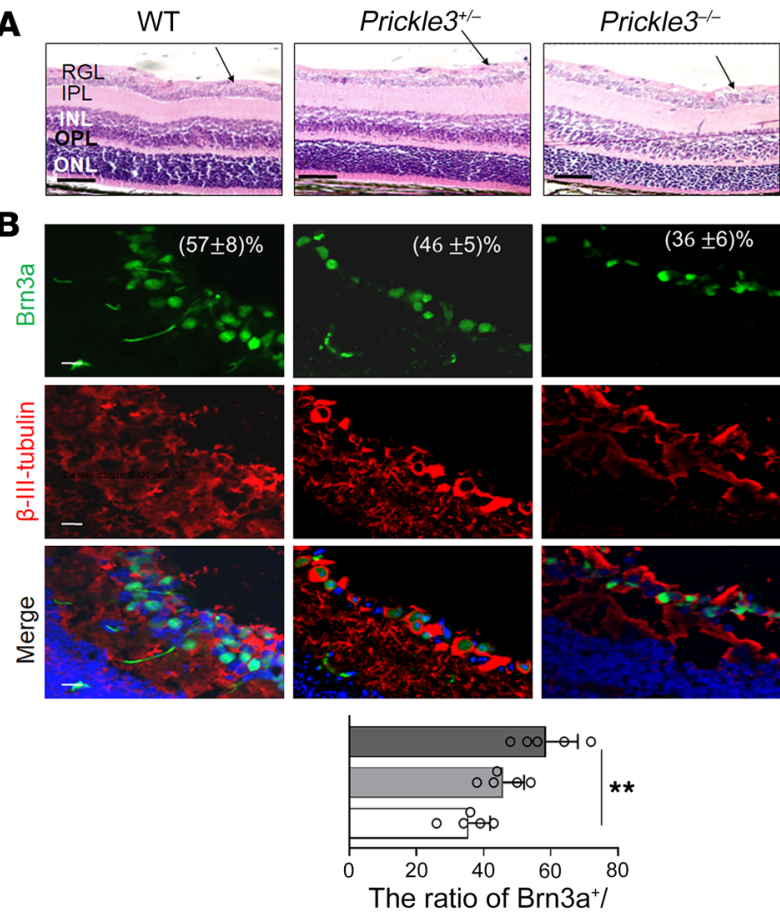

$\mathrm{DAPI}^{+}$cells $(\%)$

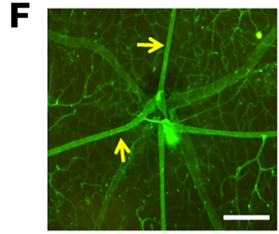

WT

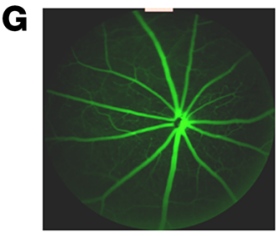

WT

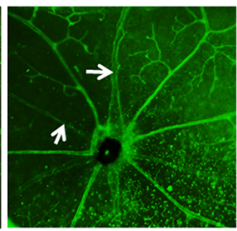

Prickle3 $^{+/-}$

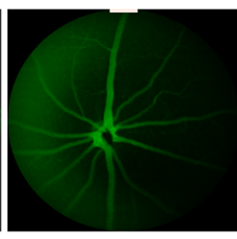

Prickle3 $^{+/}$

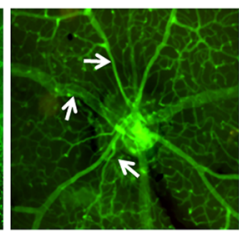

Prickle3 ${ }^{-1-}$

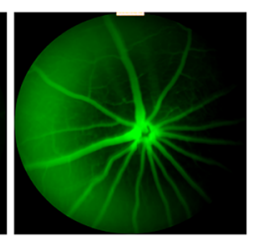

Prickle3 $^{-1}$
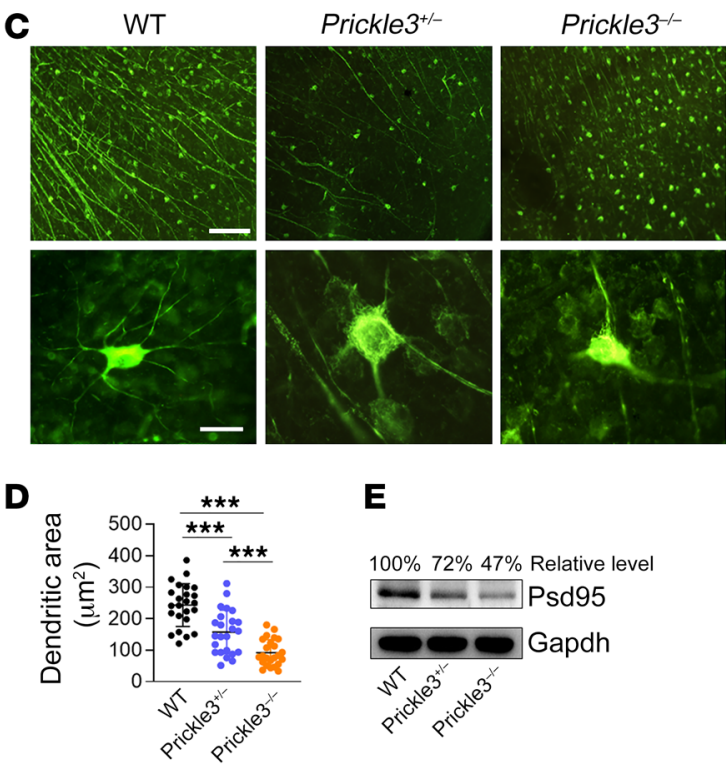

E

100\% $72 \%$ 47\% Relative level - - - Psd95

Gapdh
H
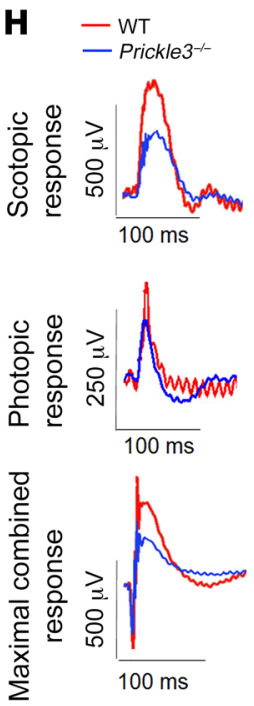
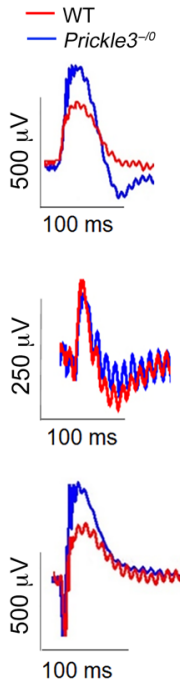
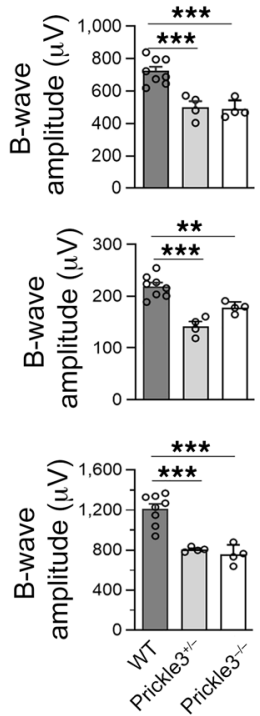

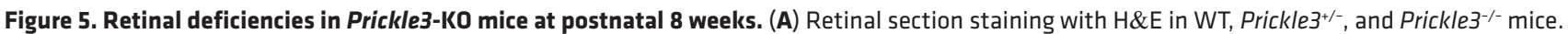
Arrows indicate RGC layer. Scale bar: $100 \mu \mathrm{m}$. (B) RGC staining with Brn3a (green) with $\beta$-III-tubulin (red) and DAPI (blue). The ratios of Brn3a+/DAPI+ cells in the RGC layer of WT $(n=5)$, Prickle3 ${ }^{+-}(n=5)$, and Prickle3 ${ }^{-/-}(n=5)$ were $57 \% \pm 8 \%, 46 \% \pm 5 \%$, and $36 \% \pm 6 \%$ from 5 crossretinal sections for each genotypic mouse sample (lower panel), respectively. Scale bar: $20 \mu \mathrm{m}$. (C) Neurofilament staining of the whole-mount retina with anti-NF-H antibody and the neurons (NF positive) with their dendrites at higher magnification. Scale bars: $200 \mu \mathrm{m}$ (upper panel), $50 \mu \mathrm{m}$ (lower panel). (D) The mean dendritic areas

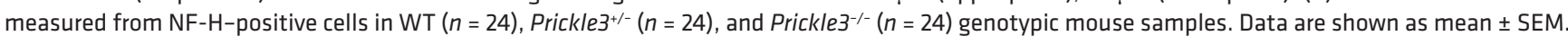
${ }^{* * *} P<0.001$ by 1-way ANOVA followed by Bonferroni's post hoc test. (E) Expression of postsynaptic density-95 (Psd95) in WT, Prickle ${ }^{+/-}$, and Prickle3-1retinas. (F) Retinal vasculature labeling with isolectin B4. Yellow arrow indicate the vessels in WT mice, and white arrows point to tortuous vessels in

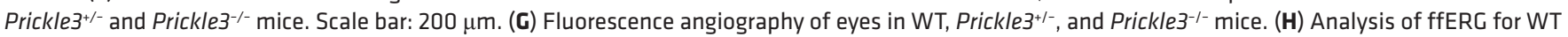
$(n=8)$, Prickle ${ }^{-/-}(n=4)$, or Prickle3-10 $(n=4)$ mice. By dark adaptation for a night, mice were analyzed for scotopic response and then photopic response. Data are shown as mean \pm SEM of triplicates. ${ }^{* *} P<0.01 ;{ }^{* *} P<0.001$ by Student's $t$ test.

To assess whether the deletion of Prickle3 altered the function of RGCs, we examined the neurofilament network and RGC dendrites on the retina by whole retinal flat-mount staining using neurofilament heavy (NF-H) (neurofilament marker) antibody (41). Both Prickle $3^{+/-}$and Prickle3 ${ }^{-/-}$mice exhibited many fewer neurofilaments in retinas than WT mice exhibited (Figure 5C). These results suggested that the loss of Prickle 3 altered the ramification of RGCs. To test whether Prickle3 deficiency caused the RGC dendropathy, we measured the body areas and dendritic areas of 100 NF-H-positive cells. As shown in Figure 5D, the average total dendritic areas on RGCs in Prickle $3^{+/-}$and Prickle $3^{-/-}$mice were $38 \%$ and $66 \%$ of those in WT mice, respectively. By contrast, the body areas of RGCs in Prickle $^{+/-}$and Prickle ${ }^{-/-}$mice were comparable with those in WT mice. The RGC dendritic pruning was further 
supported by Western blot analysis using Psd95 (a postsynaptic protein) antibody (42). As shown in Figure 5E, Psd95 levels of retinas in the Prickle $3^{+/-}$and Prickle $3^{-/-}$mice were $72 \%$ and $47 \%$ of those in the WT mice, respectively.

To examine whether the mutant mice exhibited the same vascular abnormalities in the retina as patients with $\operatorname{LHON}(43,44)$, we analyzed the retinal vasculature in Prickle $3^{+-}$, Prickle $3^{-/}$, and WT mice at the age of 8 weeks by labeling retinas with isolectin B4 (a marker for vasculature) (45). As shown in Figure 5F, the vasculature in the Prickle $^{+/-}$and Prickle3 ${ }^{-/-}$retinas was tortuous and dilated, with more branches in vessels, as compared with those in WT mice. Fluorescein angiography showed no signs of fluorescein leakage in both eyes (Figure 5G), in agreement with pathological changes seen in patients with LHON $(43,44)$. These data demonstrated that the Prickle3-KO mouse recapitulated the typical features of clinical phenotypes in patients.

Retinal functions in the Prickle $3^{-/-}$, Prickle $3^{-/ 0}$, Prickle $^{+/-}$, and WT mice were assessed by ffERG, focusing on photoreceptor deficits. As shown in Figure $5 \mathrm{H}$, the amplitudes of b-waves for scotopic (rod) responses, photopic (cone) responses, and maximal combined rod-cone responses of Prickle $3^{+-}$, Prickle $3^{-/-}$, and Prickle $^{-/ 0}$ mouse eyes at the age of 8 weeks were significantly declined, ranging from $24 \%$ to $34 \%$, as compared with WT mice. These results suggested that the photoreceptor deficits or the ERG phenotypes were the specific manifestations of RGCs, caused by the deletion of Prickle3.

\section{Discussion}

$\mathrm{X}$-linked nuclear modifier genes were proposed to increase the penetrance and expressivity of optic atrophy in families with LHON carrying the mtDNA mutations (19-21). Using the whole-exome sequencing approach, in combination with functional assays and an animal disease model, we identified the X-linked LHON susceptibility allele (c.157C>T, p.Arg53Trp) in the PRICKLE3 gene encoding a highly conserved mitochondrial protein for the phenotypic expression of the $\mathrm{m} .11778 \mathrm{G}>\mathrm{A}$ mutation in 3 Chinese families. PRICKLE3 localizes at Xp11.23, which was the putative modifier locus to modulate the phenotypic expression of $\operatorname{LHON}(20,21)$. Here, we demonstrated that the LHON phenotype was manifested by both m.11778G>A mutation and PRICKLE3 p.Arg53Trp mutation in hemizygosity in 9 men and heterozygosity in 14 women of 36 matrilineal relatives among these Chinese families. However, 2 men and 2 women carrying only a hemizygous or heterozygous p.Arg53Trp mutation did not develop the LHON phenotype, suggesting that the p.Arg53Trp mutation was by itself insufficient to produce a clinical phenotype, as in the case of YARS2 p.Gly191Val mutation (15). Furthermore, the homozygous p.Arg53Trp mutation was not detected in any women carrying the m.11778G $>$ A mutation in these Chinese and Caucasian families. Strikingly, 3 Chinese families bearing both $\mathrm{m} .11778 \mathrm{G}>\mathrm{A}$ and p.Arg53Trp mutations exhibited much higher penetrance (66\%) and younger age at onset (16 years) of optic neuropathy than those in families harboring only an LHON-associated mtDNA mutation $(8-11,17,18,46)$. In these Chinese families, there was no significant difference in average age at onset of LHON between male and female matrilineal relatives, while the affected women exhibited relatively more severe vision impairment than the affected men. Therefore, our data strongly suggested that the PRICKLE3 mutation acted as an X-linked dominant susceptible allele increasing the penetrance and expressivity of LHON-associated mtDNA mutation. This suggestion was further supported by an approximately 50\% decrease in the levels of PRICKLE3 in mutant cell lines bearing either heterozygous or hemizygous PRICKLE3 mutation. However, this p.Arg53Trp mutation is a rare variant responsible for only a very small fraction of the male preponderance observed in LHON patients overall.

In this study, we demonstrated that the PRICKLE3 protein is directly linked to the biogenesis of ATP synthase by specifically binding to the ATP8 (A6L) subunit. Human ATP synthase/complex $\mathrm{V}$ consists of $\mathrm{F}_{\mathrm{O}}$ and $\mathrm{F}_{1}$ domains, connected by 2 stalks $(36,37)$. The $\mathrm{F}_{1}$ domain is composed of subunits $\alpha, \beta, \gamma, \delta$, and $\varepsilon$, encoded by ATP5A, ATP5B, ATP5C, ATP5D, and ATP5E, respectively, while the $\mathrm{F}_{\mathrm{O}}$ domain consists of 8 subunits and a transmembrane ring, including ATP6 and ATP8 $(33,34)$. The biogenesis of complex V requires several proteins involved in $\mathrm{F}_{1}$ and $\mathrm{F}_{\mathrm{O}}$ assemblies, including ATPAF1, ATPAF2, and TMEM70 (47-49). In this study, cell lines carrying the $\mathrm{p}$.Arg53Trp mutation exhibited marked decreases in the levels of ATP6, ATP8, ATPAF1, and ATP5B. In fact, ATPAF1 bound specifically to ATP5B $(\beta)$ as an assembly factor for $\mathrm{F}_{1}(35)$, and ATP8 bound with ATP6 as a stator for the assembly of the peripheral stalk and $\mathrm{F}_{1}$ module $(36,37)$. Here, PRICKLE3 and ATP8 (A6L) reciprocally immunoprecipitated, suggesting the interaction of PRICKLE3 with ATPase by specifically binding to ATP8 (A6L). Therefore, the p.Arg53Trp mutation may alter assembling and stabilizing ATP synthase and finally lead to ATP synthase deficiency. In this study, mutant cell lines bearing the p.Arg53Trp mutation or PRICKLE3-silenced HeLa cells exhibited marked decreases in the activity and specific contents of ATP synthase. In particular, the p.Arg53Trp mutation alone conferred approximately $40 \%$ reductions in the levels of mitochondrial ATP, as in the cases of cells carrying the ATPAF2 and TMEM7O mutations $(50,51)$. However, this reduced level of mitochondrial ATP in mutant cell lines carrying only the p.Arg53Trp mutation is above a proposed threshold level, which is $50 \%$ of control levels in the mitochondrial ATP, to develop a clinical phenotype $(52,53)$, indicating that the deficient ATP production because of the $\mathrm{p}$.Arg53Trp mutation was by itself insufficient to produce the LHON phenotype. In the present study, an approximately $51 \%$ decrease in mitochondrial ATP production in the mutant cell lines derived from affected matrilineal relatives resulted from the combination of defective ATPase activity, caused by the p.Arg53Trp mutation, with $\mathrm{m} .11778 \mathrm{G}>\mathrm{A}$ mutation-induced complex I deficiency. Strikingly, approximately $50 \%$ reductions of PRICKLE3 were correlated with $50 \%$ reductions in ATPase activities in mutant cell lines bearing a heterozygous or hemizygous PRICKLE3 mutation. These reductions strongly suggested that the PRICKLE3 p.Arg53Trp mutation is a haploinsufficient mutation that acted in synergy with $\mathrm{m} .11778 \mathrm{G}>\mathrm{A}$ mutation and then deteriorated the mitochondrial dysfunctions necessary for the expression of LHON (54).

The lack of an LHON-associated ND4 mutation animal model makes it difficult to elucidate the pathogenic mechanism underlying the interaction between the ND4 and Prickle3 mutations that manifests the optic neuropathy in vivo. Therefore, we investigated biochemical and pathological consequences of Prickle3 defects in retinas using the Prickle3-KO mouse model. Prickle3-KO mice 
recapitulated the biochemical phenotypes in patients with LHON. In particular, the pronounced deficiencies of ATPase observed in Prickle3-KO mice were consistent with those in patient cell lines carrying both p.Arg53Trp and m.11778G $>$ A mutations. In contrast with the moderate ATPase deficiency in human cell lines carrying only the p.Arg53Trp mutation, the pronounced decreases in the ATPase activity caused by the deletion of Prickle 3 are likely sufficient to produce the LHON-like phenotype, including the degeneration of RGCs, abnormal vasculature, and optic nerve dysfunction $(3,4,55,56)$. Prickle3-KO mice exhibited substantial decreases in RGCs in retina ganglion layers, as in the case of the loss of RGCs in Nduf4-KO mice (57). Strikingly, the presence of many fewer neurofilaments in retinas of Prickle3-KO mice than those in WT littermates implied the altered ramification of RGCs. These Prickle3 deficiency-induced defects in the planar cell polarity of RGCs were in agreement with the known functions of Prickle family proteins $(24-26,58)$. Furthermore, Prickle3-KO mice exhibited RGC dendropathy, as evidenced by marked reductions in the dendritic areas in the NF-H-positive cells and drastic decrease of Psd95 levels in the retina, as in OPA1-KO mice (59). Moreover, Prickle3-KO mice exhibited abnormal vasculature, such as dilated and tortuous retinal vessels, with more branches, but no signs of fluorescein leakage in both eyes, consistent with pathological changes seen in patients with LHON $(1,44,45)$. Finally, Prickle3-KO mice displayed retinal deficiencies, including reductions in the dark-adapted amplitude of b-waves for scotopic response, photopic response for cone function, and maximal combined rod-cone responses of Prickle3-KO mouse eyes. These retinal deficiencies in the Prickle3-KO mice were comparable with those in patients with LHON and the mouse mtDNA-mutant model of LHON $(3,4,18$, 56). In particular, biochemical and retinal deficiencies observed in Prickle $^{+/-}$mice confirmed the haploinsufficiency of Prickle 3 mutation. Therefore, we concluded that the Prickle3-KO mouse model recapitulated the clinical phenotypes in patients with LHON.

In summary, we identified an X-linked nuclear modifier gene, PRICKLE3, for the phenotypic manifestation of LHON-associated mtDNA mutations. PRICKLE3 is directly linked to biogenesis of ATPase, and mutated PRICKLE3 altered the assembly, stability, and function of ATP synthase. The PRICKLE3 p.Arg53Trp is a haploinsufficient mutation that acted in synergy with $\mathrm{m} .11778 \mathrm{G}>\mathrm{A}$ mutation and then deteriorated the mitochondrial dysfunctions necessary for the expression of LHON. The Prickle3-KO mouse model exhibited pronounced decreases in the content of fully assembled and functional ATP synthase, consistent with those in patients with LHON. Prickle3-KO mice recaptured LHON phenotypes with the associated retinal deficiencies. Our findings provide new insights into the pathophysiology of LHON that was manifested by interactions between ND4 and X-linked PRICKLE3 mutations. Furthermore, the approach used in this study provides a paradigm for understanding the role of nuclear modifier genes in the pathogenic effects of mtDNA mutations and the mitochondrial functions in RGCs.

\section{Methods}

See additional information in Supplemental Methods.

Families and subjects. DNA samples used for this investigation were from 361 symptomatic and 234 asymptomatic members of 211
Han Chinese families, 58 symptomatic and 58 asymptomatic individuals from 58 Caucasian pedigrees carrying the m.11778G $>$ A mutation, and 1515 Han Chinese and 237 Caucasian control subjects lacking the m.11778G $>$ A mutation $(11,22,23,27,60)$. The ophthalmic examinations and other clinical evaluations of probands, other members of these families, and control subjects were conducted as detailed elsewhere $(23,27)$. Informed consent, blood samples, and clinical evaluations were obtained from all participants and families, under protocols approved by the Ethic Committee of Zhejiang University School of Medicine and Institutional Review Board of Cincinnati Children's Hospital Medical Center.

Sequencing and genetic data analysis. Whole-exome sequencings of 4 members (III-14, III-15, II-7, and II-8) of the SD1 pedigree were performed by BGI. The data for these whole-exome sequences were submitted into the National Center for Biotechnology Information's BioProject database (accession PRJNA634625). High-quality genomic DNA $(3 \mu \mathrm{g})$ was captured by hybridization using the SureSelect XT Human All Exon 50Mb kit (Agilent Technologies). Samples were prepared according to the manufacturer's instructions. Each captured library was run on a HiSeq 2000 instrument (Illumina), and sequences were generated as $90 \mathrm{bp}$ paired-end reads. On average, 82 million paired reads were generated per sample, the mean duplication rate was $13.45 \%$, and $98.64 \%$ of targeted regions were covered by at least 70 times mean depth. All sequencing reads were mapped to the human reference genome (GRCh37) at UCSC Genome Browser. SOAPsnp software was used to assemble the consensus sequence and call genotypes in the target regions. GATK (IndelGenotyper V1.0) was used for indel detection. The threshold for filtering SNPs included the following criteria: SNP quality score should be at least 20, sequencing depth should be between 4 and 200, estimated copy numbers should be no more than 2, and the distance between 2 SNPs should be larger than 5. SNPs from these analyses are summarized in Supplemental Table 2. Variants were annotated by ANNOVAR software. To further filter the SNPs, the criteria for potential candidate variants were nonsynonymous or in splice sites within 6 bp of an exon, less than $1 \%$ mutant allele frequency in variant databases, and cosegregated with the phenotype. The mutations were validated by Sanger sequencing in all family members and samples from nonrelatives.

Primers of PRICKLE3 for Sanger sequencing are listed in Supplemental Table 3, including the primers for the genotyping of the c.157C > T mutation in exon 3. The entire mtDNA of 3 Chinese families carrying both PRICKLE3 c.157C > T and m.11778G $>$ A mutations was analyzed as described elsewhere (29). The resulting sequences were compared with the updated consensus Cambridge sequence (GenBank accession number NC_012920) (6). The copy numbers of mtDNA from mutant and control cell lines were determined as described elsewhere (61).

Generation and genotyping of Prickle3-KO mice. All animal care protocols were approved by the Animal Care and Use Committee of Zhejiang University School of Medicine. C57BL/6J mice were originally purchased from Shanghai SLAC Laboratory Animal Co, Ltd. Sanger sequencing analysis of the $\mathrm{Crb} 1$ gene failed to detect the Rd8 mutation in vendor lines of C57BL/6 J mice (62). Prickle3-KO mice were generated using the CRISPR/Cas9 approach. The targeting genomic RNA and donor oligonucleotides for Prickle3 are listed in Supplemental Table 3. Cas9 mRNA, genomic RNAs, and donor vector were mixed at different concentrations and coinjected into the cytoplasm of fertilized eggs at the 1-cell stage. After injection, surviving zygotes were transferred 
into the oviducts of pseudopregnant females. The genotypes for the Prickle ${ }^{+/-}$, Prickle3 ${ }^{-/}$, and Prickle3 $3^{-/ 0}$ mice were confirmed by PCR amplification and direct sequencing. Mice bearing the Prickle3 $13 \mathrm{bp}$ deletion was selected and then mated with C57BL/6J female mice.

Cell lines and culture conditions. Lymphoblastoid cell lines were generated from members of the SD1 pedigree (2 affected individuals, II- 8 and III-2, carrying both $\mathrm{m} .11778 \mathrm{G}>\mathrm{A}$ and heterozygous c.157C $>$ T mutations; 2 affected members, III-13 and III-14, carrying both m.11778G $>$ A and hemizygous c.157C $>$ T mutations; 2 normalvision subjects, II-9 and III-15, carrying only m.11778G $>$ A mutation; 1 individual, IV-3, carrying only a heterozygous c.157C $>$ T mutation; and 2 genetically unrelated control subjects, C1-2 and WZ209, lacking both mutations), as detailed previously (63). Lymphoblastoid cell lines were grown in RPMI 1640 medium (Invitrogen, Thermo Fisher Scientific), supplemented with $10 \%$ fetal bovine serum (FBS). HeLa and HEK293T cell lines (ATCC) were grown in DMEM (Corning Inc.), supplemented with $10 \%$ FBS.

Western blot and immunoprecipitation assays. Western blot analysis was performed using $20 \mu \mathrm{g}$ of total cellular proteins isolated from human cell lines or mouse tissues, as detailed elsewhere $(15,64)$. The antibodies used for this investigation are summarized in Supplemental Table 5. Peroxidase AffiniPure goat anti-mouse IgG and goat anti-rabbit IgG (Beyotime A0216 and A0208, respectively) were used as secondary antibodies, and protein signals were detected using the ECL system (CWBIO). The quantification of density in each band was performed as detailed previously $(15,64)$.

For the immunoprecipitation (IP) analysis, mitochondria were extracted from the HEK293T cell line after transfection with PRICKLE3-HA for 36 hours as described elsewhere (65). Five micrograms of mitochondrial proteins were solubilized in $1 \mathrm{~mL}$ PBS containing $0.5 \%$ N-Dodecyl- $\beta$-D-maltoside (DDM; MedChemExpress, HY-128974) on ice for 30 minutes and centrifuged at 20,000 $\mathrm{g}$ for 10 minutes at $4^{\circ} \mathrm{C}$. The supernatants were incubated with $10 \mu \mathrm{L}$ of beads (cross-linked to $25 \mu \mathrm{g}$ of monoclonal antibody, anti-HA, or anti-ATPase complex) overnight at $4^{\circ} \mathrm{C}$ with rotation. Beads were washed 4 times, then boiled for 5 minutes after SDS loading was added. Finally, the IP fractions were analyzed by Western blot analysis.

Enzymatic activity assays. The enzymatic activities of OXPHOS complexes I, II, III, IV, and V were measured as detailed elsewhere $(66,67)$. For evaluating the activity of complex V in gel, $20 \mu \mathrm{g}$ total cellular protein samples from human cell lines or mouse tissues were loaded and run at $150 \mathrm{~V}$ in light-blue cathode buffer for 1 hour, then at $250 \mathrm{~V}$ in clear cathode buffer. The native gels were prewashed in cold water and then incubated with the substrates of complex $\mathrm{V}$ in a buffer containing $35 \mathrm{mM}$ Tris, $270 \mathrm{mM}$ glycine, $14 \mathrm{mM} \mathrm{MgSO}_{4}, 10 \mathrm{mM}$ ATP, and $0.2 \% \mathrm{~Pb}\left(\mathrm{NO}_{3}\right)_{2}$ at room temperature overnight. The gel was washed with $50 \%$ methanol 3 times. Images were captured and quantified as described elsewhere (37).

Measurements of oxygen consumption. The rates of oxygen consumption in mutant and control cell lines were measured with a Seahorse Bioscience XFe96 extracellular flux analyzer, as detailed elsewhere (68-70).

ATP measurements. The CellTiter-Glo Luminescent Cell Viability Assay kit (Promega) was used for the measurement of cellular and mitochondrial ATP levels, according to the manufacturer's instructions with some modification $(65,68)$.

Stability analysis of ATPase by Native PAGE. The stability of assembled ATPase was analyzed by mitochondria isolated from human cell lines and mouse livers using blue native gel electrophoresis $(31,32)$. In brief, $400 \mu \mathrm{g}$ mitochondria were solubilized in $0.5 \%$ DDM solution containing $50 \mathrm{mM} \mathrm{NaCl}, 50 \mathrm{mM}$ imidazole, $2 \mathrm{mM}$ 6-aminohexanoic acid, and $1 \mathrm{mM}$ EDTA ( $\mathrm{pH}$ 7.4) on ice for 20 minutes. After removing insoluble material by centrifugation, $10 \mu \mathrm{g}$ of lysed mitochondria from each sample were loaded onto 3\%-12\% gradient Native PAGE Bis-Tris gels (Thermo Fisher Scientific) and run at $150 \mathrm{~V}$ in dark-blue cathode buffer for 1 hour and then $250 \mathrm{~V}$ in light-blue running buffer for 1.5 hours at $4^{\circ} \mathrm{C}$. The native gels were then prewashed in a transfer buffer $(25 \mathrm{mM}$ Tris, $192 \mathrm{mM}$ glycine, 20\% methanol) and transferred to the polyvinylidene fluoride membrane at $30 \mathrm{~V}$ overnight for immunoblotting.

Immunofluorescence and immunohistochemistry. For NF-H flatmount staining, whole mouse retinas (at 8 weeks) were fixed in $4 \%$ paraformaldehyde at $4^{\circ} \mathrm{C}$ for 2 hours, permeabilized with $0.2 \%$ Tween for 1 hour, blocked with 5\% FBS serum buffer $(+0.2 \%$ Triton $\mathrm{X}-100$ in PBS) at room temperature for 1 hour, and incubated with mouse anti-NF-H (1:1000, Cell Signaling Technology, 2836) at $4^{\circ} \mathrm{C}$ overnight and then secondary antibody, Alexa Fluor 488 goat anti-mouse IgG (Abcam, ab150113), for 2 hours. Retinas were washed again 3 times for 3 hours and mounted in a mounting medium. Images were taken by Leica DM4000B-M. Nikon NIS-Elements imaging software was used to automatically measure cell body area and dendritic field in the RGCs as described previously $(41,71)$.

Cross sections of retinal staining with Brn3a and tubulin were performed as detailed previously $(38,39)$. Eye cups were dissected and immersed in 4\% paraformaldehyde in PBS for 24 hours, then embedded in O.C.T. compound (Thermo Fisher Scientific) after infiltrating with $30 \%$ sucrose overnight. Transverse sections of the retina $(10 \mu \mathrm{m}$ thick) were mounted onto slides and blocked with $20 \%$ fetal calf serum in PBS for 1 hour. The retinas were incubated with primary antibodies, anti-Brn3a (1:500, Abcam ab81223) and anti- $\beta$-III-tubulin (1:2500, Abcam ab18207) at $4^{\circ} \mathrm{C}$ overnight, and then the secondary antibodies, Alexa Fluor 488 goat anti-mouse IgG and Alexa Fluor 594 goat anti-rabbit IgG. Images were taken by Leica DM4000B-M and Olympus Fluoview FV1000 microscopes with DAPI for staining of nuclei.

Fluorescein angiography. Fluorescence angiography of eyes was carried out in Prickle3-KO and WT mice (at 8 weeks) using the Micron III camera (Phoenix Research Laboratories, Inc.) as described previously (72). Pupils were dilated with $1 \%$ tropicamide (Bausch \& Lomb) followed by application of GenTeal Lubricant Eye Gel (Alcon). Systane lubricant eye drops (Alcon) were applied to keep the cornea moist. Mouse pupils were then intraperitoneally injected with 25\% Angiofluor (Alliance Pharma) at a dose of $0.01 \mathrm{~mL}$ per $5 \mu \mathrm{g}$ of mouse body weight. Photos were taken with a camera containing a barrier filter for fluorescein angiography.

Retinal vessel labeling. Whole mouse retinas were fixed for half an hour and blocked with $5 \%$ FBS serum buffer at $4^{\circ} \mathrm{C}$ overnight, then equilibrated with a solution $\left(1 \mu \mathrm{M} \mathrm{MgCl}_{2}, 1 \mu \mathrm{M} \mathrm{CaCl}_{2}, 0.1 \mu \mathrm{M} \mathrm{MnCl}_{2}\right.$, $0.1 \%$ Triton X-100 in PBS) for 1 hour at room temperature. After incubating with FITC-conjugated isolectin B4 (1:50 in blocking buffer, MilliporeSigma) at $4^{\circ} \mathrm{C}$ overnight, the retinas were washed, mounted, and then imaged by Leica DM4000B-M.

ERG measurement. ERGs of mice (at 8 weeks) were recorded as previously described (41). Both the scotopic and photopic ERGs were recorded with a well-established Ganzfeld Q450 dome stimulating and recording system. Mice were dark-adapted and anesthetized with $\mathrm{Ag} / \mathrm{AgCl}$ wire loop electrode placed over the cornea. 
Statistics. Statistical analysis was performed using GraphPad Prism (version 8.0.2) for statistical analysis to compare outcomes using a 2-tailed paired or unpaired Student's $t$ test. For multiple comparisons, 1-way ANOVA or 2-way ANOVA was performed. $P$ values of less than 0.05 were considered statistically significant.

Study approval. Informed consent in writing before their participation in this study was obtained from members of families and control subjects, under protocols approved by the Ethic Committee of Zhejiang University School of Medicine and Institutional Review Board of Cincinnati Children's Hospital Medical Center. Furthermore, all experiments involving mice were approved by the Institutional Animal Care and Use Committee, Zhejiang University School of Medicine.

\section{Author contributions}

MXG and PJ designed the experiments and monitored the project progression, data analysis, and data interpretation. JY, JL, and CA performed the biochemical analyses; JY, YJ, JL, LZ, XJ, and $\mathrm{X}$ Zhao performed the whole-exome sequencing and mutational screening; XL, JY, CA, ZN, CW, and SM carried out the mouse experiments; YJ, JZ, FZ, X Zhou, MZ, and TH carried out the clinical evaluation and recruited patients with LHON; JY and
MW performed the data analysis; and PJ prepared the initial draft of the manuscript. MXG made the final version of the manuscript. All authors reviewed the manuscript.

\section{Acknowledgments}

We are grateful to the patients and their family members for their participation. This work was supported by National Key Research and Development Program of China grant 2018YFC1004802 to MXG; grants 31671303, 31970557, and 81900904 from the National Natural Science Foundation of China to PJ, MXG, and JY, respectively; the National Key Technologies R\&D Program of China grant 2012BAIO9B03 to MXG and PJ; and the Zhejiang Provincial Program for the Cultivation of High-level Innovative Health Talents to PJ.

Address correspondence to: Min-Xin Guan, Institute of Genetics, Zhejiang University School of Medicine, 866 Yuhangtang Road, Hangzhou, Zhejiang 310058, China. Phone: 86.571.88206916; Email: gminxin88@zju.edu.cn. Or to: Pingping Jiang, Department of Human Genetics, Zhejiang University School of Medicine, 866 Yuhangtang Road, Hangzhou, Zhejiang 310058, China. Phone: 86.571.88208328; Email:ppjiang@zju.edu.cn.
1. Wallace DC, Lott MT. Leber hereditary optic neuropathy: exemplar of an mtDNA disease. Handb Exp Pharmacol. 2017;240:339-376.

2. Howell N. Leber hereditary optic neuropathy: respiratory chain dysfunction and degeneration of the optic nerve. Vision Res. 1998;38(10):1495-1504.

3. Sadun AA, La Morgia C, Carelli V. Leber's hereditary optic neuropathy. Curr Treat Options Neurol. 2011;13(1):109-117.

4. Newman NJ. Hereditary optic neuropathies: from the mitochondria to the optic nerve. Am JOphthalmol. 2005;140(3):517-523.

5. Carelli V, La Morgia C, Valentino ML, Barboni P, Ross-Cisneros FN, Sadun AA. Retinal ganglion cell neurodegeneration in mitochondrial inherited disorders. Biochim Biophys Acta. 2009;1787(5):518-528.

6. Andrews RM, Kubacka I, Chinnery PF, Lightowlers RN, Turnbull DM, Howell N. Reanalysis and revision of the Cambridge reference sequence for human mitochondrial DNA. Nat Genet. 1999;23(2):147.

7. Wallace DC, et al. Mitochondrial DNA mutation associated with Leber's hereditary optic neuropathy. Science. 1988;242(4884):1427-1430.

8. Ruiz-Pesini E, et al. An enhanced MITOMAP with a global mtDNA mutational phylogeny. Nucleic Acids Res. 2007;35(Database issue):D823-D828.

9. Brown MD, Torroni A, Reckord CL, Wallace DC. Phylogenetic analysis of Leber's hereditary optic neuropathy mitochondrial DNA's indicates multiple independent occurrences of the common mutations. Hum Mutat. 1995;6(4):311-325.

10. Yu-Wai-Man P, Griffiths PG, Hudson G, Chinnery $\mathrm{PF}$. Inherited mitochondrial optic neuropathies. JMed Genet. 2009;46(3):145-158.

11. Jiang $P$, et al. Prevalence of mitochondrial ND4 mutations in 1281 Han Chinese subjects with Leber's hereditary optic neuropathy. Invest Ophthalmol Vis Sci. 2015;56(8):4778-4788.
12. Mashima Y, et al. Spectrum of pathogenic mitochondrial DNA mutations and clinical features in Japanese families with Leber's hereditary optic neuropathy. Curr Eye Res. 1998;17(4):403-408.

13. Brown MD, Trounce IA, Jun AS, Allen JC, Wallace DC. Functional analysis of lymphoblast and cybrid mitochondria containing the 3460,11778 , or 14484 Leber's hereditary optic neuropathy mitochondrial DNA mutation. J Biol Chem. 2000;275(51):39831-39836.

14. Hofhaus G, Johns DR, Hurko O, Attardi G, Chomyn A. Respiration and growth defects in transmitochondrial cell lines carrying the 11778 mutation associated with Leber's hereditary optic neuropathy. J Biol Chem. 1996;271(22):13155-13161.

15. Jiang $P$, et al. The exome sequencing identified the mutation in YARS2 encoding the mitochondrial tyrosyl-tRNA synthetase as a nuclear modifier for the phenotypic manifestation of Leber's hereditary optic neuropathy-associated mitochondrial DNA mutation. Hum Mol Genet. 2016;25(3):584-596.

16. Qian Y, Zhou X, Liang M, Qu J, Guan MX. The altered activity of complex III may contribute to the high penetrance of Leber's hereditary optic neuropathy in a Chinese family carrying the ND4 G11778A mutation. Mitochondrion. 2011;11(6):871-877.

17. Newman NJ, Lott MT, Wallace DC. The clinical characteristics of pedigrees of Leber's hereditary optic neuropathy with the 11778 mutation. Am J Ophthalmol. 1991;111(6):750-762.

18. Riordan-Eva P, Sanders MD, Govan GG, Sweeney MG, Da Costa J, Harding AE. The clinical features of Leber's hereditary optic neuropathy defined by the presence of a pathogenic mitochondrial DNA mutation. Brain. 1995;118(pt 2):319-337.

19. Bu XD, Rotter JI. X chromosome-linked and mitochondrial gene control of Leber hereditary optic neuropathy(18): evidence from segregation analysis for dependence on X chromo- some inactivation. Proc Natl Acad Sci US A. 1991;88(18):8198-8202.

20. Hudson G, et al. Identification of an X-chromosomal locus and haplotype modulating the phenotype of a mitochondrial DNA disorder. Am J Hum Genet. 2005;77(6):1086-1091.

21. Shankar SP, et al. Evidence for a novel $\mathrm{x}$-linked modifier locus for leber hereditary optic neuropathy. Ophthalmic Genet. 2008;29(1):17-24.

22. Ji Y, et al. Mitochondrial ND1 variants in 1281 Chinese subjects with Leber's hereditary optic neuropathy. Invest Ophthalmol Vis Sci. 2016;57(6):2377-2389.

23. Liang M, et al. Frequency and spectrum of mitochondrial ND6 mutations in 1218 Han Chinese subjects with Leber's hereditary optic neuropathy. Invest Ophthalmol Vis Sci. 2014;55(3):1321-1331.

24. Collu GM, et al. Prickle is phosphorylated by Nemo and targeted for degradation to maintain Prickle/Spiny-legs isoform balance during planar cell polarity establishment. PLoS Genet. 2018;14(5):e1007391.

25. Ehaideb SN, et al. prickle modulates microtubule polarity and axonal transport to ameliorate seizures in flies. Proc Natl Acad Sci U S A. 2014;111(30):11187-11192.

26. Ossipova O, Chu CW, Fillatre J, Brott BK, Itoh $\mathrm{K}$, Sokol SY. The involvement of PCP proteins in radial cell intercalations during Xenopus embryonic development. Dev Biol. 2015;408(2):316-327.

27. Zhou X, et al. Very high penetrance and occurrence of Leber's hereditary optic neuropathy in a large Han Chinese pedigree carrying the ND4 G11778A mutation. Mol Genet Metab. 2010;100(4):379-384.

28. Tao H, et al. Mutations in prickle orthologs cause seizures in flies, mice, and humans. Am J Hum Genet. 2011;88(2):138-149.

29. Rieder MJ, Taylor SL, Tobe VO, Nickerson DA. Automating the identification of DNA variations using quality-based fluorescence re-sequencing 
analysis of the human mitochondrial genome. Nucleic Acids Res. 1998;26(4):967-973.

30. Claros MG, Vincens P. Computational method to predict mitochondrially imported proteins and their targeting sequences. Eur J Biochem. 1996;241(3):779-786.

31. Jha P, Wang X, Auwerx J. Analysis of mitochondrial respiratory chain supercomplexes using blue native polyacrylamide gel electrophoresis (BN-PAGE). Curr Protoc Mouse Biol. 2016;6(1):1-14.

32. Wittig I, Braun HP, Schägger H. Blue native PAGE. Nat Protoc. 2006;1(1):418-428.

33. Jonckheere AI, Smeitink JA, Rodenburg RJ. Mitochondrial ATP synthase: architecture, function and pathology. J Inherit Metab Dis. 2012;35(2):211-225.

34. Junge W, Nelson N. ATP synthase. Annu Rev Biochem. 2015;84:631-657.

35. Naumenko N, Morgenstern M, Rucktäschel R, Warscheid B, Rehling P. INA complex liaises the $\mathrm{F}_{1} \mathrm{~F}_{\mathrm{o}}$-ATP synthase membrane motor modules. Nat Commun. 2017;8(1):1237.

36. Guo H, Bueler SA, Rubinstein JL. Atomic model for the dimeric $\mathrm{F}_{\mathrm{O}}$ region of mitochondrial ATP synthase. Science. 2017;358(6365):936-940.

37. He J, et al. Assembly of the membrane domain of ATP synthase in human mitochondria. Proc Natl Acad Sci U S A. 2018;115(12):2988-2993.

38. McCulloch DL, et al. ISCEV Standard for fullfield clinical electroretinography (2015 update). Doc Ophthalmol. 2015;130(1):1-12.

39. Badea TC, Cahill H, Ecker J, Hattar S, Nathans J. Distinct roles of transcription factors brn3a and brn3b in controlling the development, morphology, and function of retinal ganglion cells. Neuron. 2009;61(6):852-864.

40. Goel M, Dhingra NK. Müller glia express rhodop$\sin$ in a mouse model of inherited retinal degeneration. Neuroscience. 2012;225:152-161.

41. Song L, Yu A, Murray K, Cortopassi G. Bipolar cell reduction precedes retinal ganglion neuron loss in a complex 1 knockout mouse model. Brain Res. 2017;1657:232-244.

42. Li X, et al. Gene therapy rescues cone structure and function in the 3-month-old rd12 mouse: a model for midcourse RPE65 leber congenital amaurosis. Invest Ophthalmol Vis Sci. 2011;52(1):7-15.

43. Carelli V, La Morgia C, Ross-Cisneros FN, Sadun AA. Optic neuropathies: the tip of the neurodegeneration iceberg. Hum Mol Genet. 2017;26(R2):R139-R150.

44. Kerrison JB, Newman NJ. Clinical spectrum of Leber's hereditary optic neuropathy. Clin Neurosci. 1997;4(5):295-301.
45. Zuercher J, Fritzsche M, Feil S, Mohn L, Berger W. Norrin stimulates cell proliferation in the superficial retinal vascular plexus and is pivotal for the recruitment of mural cells. Hum Mol Genet. 2012;21(12):2619-2630.

46. Harding AE, Sweeney MG, Govan GG, Riordan-Eva P. Pedigree analysis in Leber hereditary optic neuropathy families with a pathogenic mtDNA mutation. Am JHum Genet. 1995;57(1):77-86.

47. Rak M, Gokova S, Tzagoloff A. Modular assembly of yeast mitochondrial ATP synthase. EMBO J. 2011;30(5):920-930.

48. Vrbacký M, et al. Knockout of Tmem70 alters biogenesis of ATP synthase and leads to embryonal lethality in mice. Hum Mol Genet. 2016;25(21):4674-4685.

49. Wang ZG, White PS, Ackerman SH. Atp11p and Atp12p are assembly factors for the $F(1)$ ATPase in human mitochondria.J Biol Chem. 2001;276(33):30773-30778.

50. De Meirleir L, et al. Respiratory chain complex $\mathrm{V}$ deficiency due to a mutation in the assembly gene ATP12. J Med Genet. 2004;41(2):120-124.

51. Spiegel R, et al. TMEM70 mutations are a common cause of nuclear encoded ATP synthase assembly defect: further delineation of a new syndrome. J Med Genet. 2011;48(3):177-182.

52. Houstek J, et al. A novel deficiency of mitochondrial ATPase of nuclear origin. Hum Mol Genet. 1999;8(11):1967-1974.

53. Trounce I, Neill S, Wallace DC. Cytoplasmic transfer of the mtDNA nt 8993 T--> G (ATP6) point mutation associated with Leigh syndrome into mtDNA-less cells demonstrates cosegregation with a decrease in state III respiration and ADP/O ratio. Proc Natl Acad Sci U S A. 1994;91(18):8334-8338.

54. Bartha I, di Iulio J, Venter JC, Telenti A. Human gene essentiality. Nat Rev Genet. 2018;19(1):51-62.

55. Jonckheere AI, et al. Restoration of complex $\mathrm{V}$ deficiency caused by a novel deletion in the human TMEM70 gene normalizes mitochondrial morphology. Mitochondrion. 2011;11(6):954-963.

56. Lin CS, et al. Mouse mtDNA mutant model of Leber hereditary optic neuropathy. Proc Natl Acad Sci U S A. 2012;109(49):20065-20070.

57. Yu AK, et al. Mitochondrial complex I deficiency leads to inflammation and retinal ganglion cell death in the Ndufs 4 mouse. Hum Mol Genet. 2015;24(10):2848-2860.

58. Chu CW, Sokol SY. Wnt proteins can direct planar cell polarity in vertebrate ectoderm. Elife. 2016;5:e16463.

59. Williams PA, Morgan JE, Votruba M. Opa1 defi- ciency in a mouse model of dominant optic atrophy leads to retinal ganglion cell dendropathy. Brain. 2010;133(10):2942-2951.

60. Ji Y, et al. Contribution of mitochondrial ND1 $3394 \mathrm{~T}>\mathrm{C}$ mutation to the phenotypic manifestation of Leber's hereditary optic neuropathy. Hum Mol Genet. 2019;28(9):1515-1529.

61. Zhang J, et al. A novel ADOA-associated OPA1 mutation alters the mitochondrial function, membrane potential, ROS production and apoptosis. Sci Rep. 2017;7(1):5704.

62. Mattapallil MJ, et al. The Rd8 mutation of the $\mathrm{Crb} 1$ gene is present in vendor lines of $\mathrm{C} 57 \mathrm{BL} / 6 \mathrm{~N}$ mice and embryonic stem cells, and confounds ocular induced mutant phenotypes. Invest Ophthalmol Vis Sci. 2012;53(6):2921-2927.

63. Miller G, Lipman M. Release of infectious EpsteinBarr virus by transformed marmoset leukocytes. Proc Natl Acad Sci U S A. 1973;70(1):190-194.

64. Zhou M, et al. A hypertension-associated mitochondrial DNA mutation introduces an $\mathrm{m}^{1} \mathrm{G} 37$ modification into tRNA ${ }^{\text {Met }}$, altering its structure and function. J Biol Chem. 2018;293(4):1425-1438.

65. Ausenda C, Chomyn A. Purification of mitochondrial DNA from human cell cultures and placenta. Meth Enzymol. 1996;264:122-128.

66. Birch-Machin MA, Turnbull DM. Assaying mitochondrial respiratory complex activity in mitochondria isolated from human cells and tissues. Methods Cell Biol. 2001;65:97-117.

67. Zhang J, et al. Leber's hereditary optic neuropathy (LHON)-associated ND512338T $>C$ mutation altered the assembly and function of complex I, apoptosis and mitophagy. Hum Mol Genet. 2018;27(11):1999-2011.

68. Gong S, et al. A deafness-associated tRNAHis mutation alters the mitochondrial function, ROS production and membrane potential. Nucleic Acids Res. 2014;42(12):8039-8048.

69. Dranka BP, et al. Assessing bioenergetic function in response to oxidative stress by metabolic profiling. Free Radic Biol Med. 2011;51(9):1621-1635.

70. Jiang P, et al. Biochemical evidence for a mitochondrial genetic modifier in the phenotypic manifestation of Leber's hereditary optic neuropathyassociated mitochondrial DNA mutation. Hum Mol Genet. 2016;25(16):3613-3625.

71. Leung CK, et al. Long-term in vivo imaging and measurement of dendritic shrinkage of retinal ganglion cells. Invest Ophthalmol Vis Sci. 2011;52(3):1539-1547.

72. Spaide RF, Klancnik JM, Cooney MJ. Retinal vascular layers imaged by fluorescein angiography and optical coherence tomography angiography. JAMA Ophthalmol. 2015;133(1):45-50. 